\begin{tabular}{|c|c|}
\hline \multirow{3}{*}{ 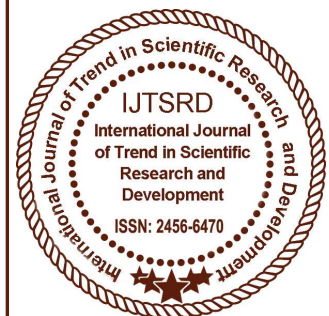 } & $\begin{array}{l}\text { International Journal of Trend in Scientific } \\
\text { Research and Development (IJTSRD) }\end{array}$ \\
\hline & International Open Access Journal \\
\hline & ISSN No: 2456 - 6470 | www.ijtsrd.com | Volume - 2 | Issue - 1 \\
\hline
\end{tabular}

\title{
Impact of Junk Foods and it's Banning among Adolescent Girls
}

\author{
Dr. Nisha Vikraman \\ Assistant Professor, Department of Home Science, \\ St. Teresa's college, Ernakulam, Kerala, India
}

\author{
Nitha \\ Department of Home Science, \\ St. Teresa's college, Ernakulam, Kerala, India
}

\begin{abstract}
India has seen a massive rise in the consumption of fast food over the recent few years. Various factors have influenced Indian market to lean towards fast food culture. The study reveals the impact and banning of fast food among adolescent girls. Majority of parents stop their children from eating fast foods. most of the subjects were aware that the fast foods are unhealthy.
\end{abstract}

Keywords: fast foods; awareness; questionnaire; adolescence; eating habit

\section{INTRODUCTION}

Fast food culture is a vigorously uprising trend among the adolescence (1). Although, its impact exits on whole society, whether belong to lower middle class and/or elite class. But beside this thing till today there is no proper definition of the fast food. According to leading websites, fast food is the term given to food that can be prepared and served very quickly while Habib et al. (2) described fast food as quickly prepared, reasonably priced and readily available alternatives to home cooked food.

Fast food industry has helped to transform not only our diet, but also economy and culture as well. Fast food has now become a regular part of Indian diet and on an average of almost one third of youths from 1419 years consumes fast food on a typical day $(3,4)$.

\section{METHODOLOGY}

\section{A. Selection of Area}

Autonomous colleges in an urban area were selected for the study. The study was conducted among the first year students of St. Teresa's autonomous college, Ernakulam.

\section{B. Selection of Method}

The method used for the study is a self-administered questionnaire method.

\section{Selection of Sample}

About 30 students were selected at random sampling from different departments in the age group between 17-19 years of age.

\section{Selection of Tool}

Self-administered questionnaire prepared by the investigator with 20 closed ended questions and 3 open ended questions was selected as the tool for the required study.

\section{E. Collection of Data}

The investigator visited the institution on September 8,2015 and the data was collected on the same day.

The observation of the collected data was done on September 13, 2015.

\section{RESULT AND DISCUSSION}

\section{A. Junk Food Eating Habit of the Students}

The eating habits of the students revealed that $60 \%$ of students did not take junk food as an alternative to breakfast while $40 \%$ took it as an alternative and when students were asked to select the factors that influences them in selecting the type of junk foods, $20 \%$ opted for taste alone while a better part for taste and appearance $13 \%$ taste and peer groups, $7 \%$ taste 
along with convenience and peer group, 13\% opted for taste and time factor.

\section{B. Awareness about junk foods}

The awareness about junk food facts showed that about $57 \%$ of respondents said that they do check the quality of the junk food followed by $43 \%$ who don't check it. For the question related to nutrient fact label awareness $40 \%$ checked while majority about $60 \%$ don't check it. In case of chemicals and their safety level information only $17 \%$ were aware about it and $83 \%$ were unaware about it. When asked about the harmful effects of chemicals present $77 \%$ were not aware about the chemical. Also $70 \%$ of them were unaware of the harmful side effects. Responses to various parameters such as quality, nutrient fact labeling, chemicals \& harmful effect.

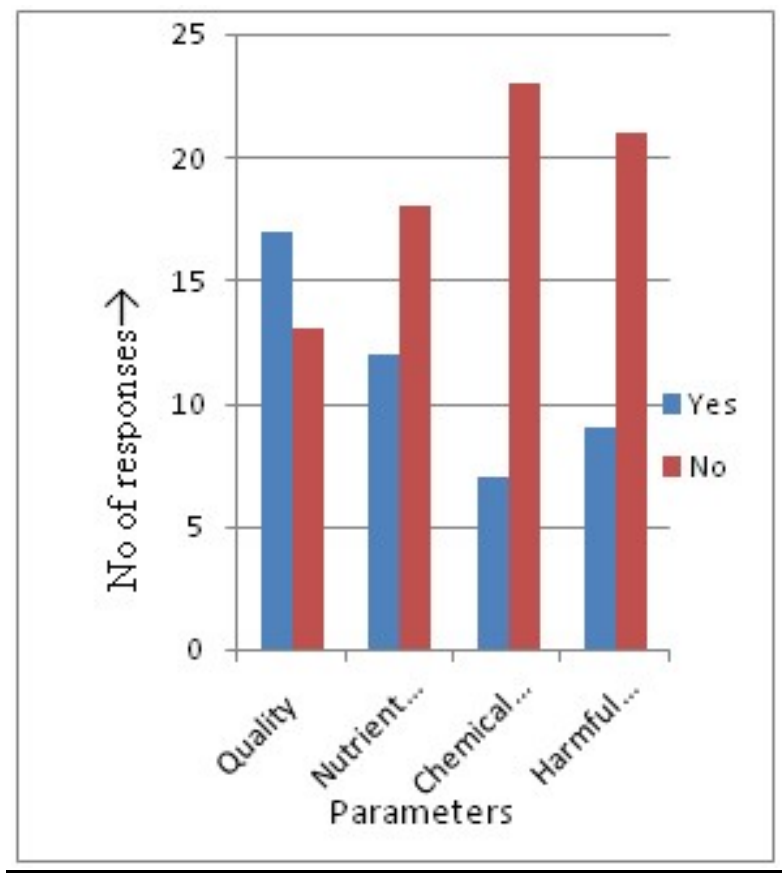

Fig. 1 awareness about junk food

\section{Opinion about junk foods}

About $87 \%$ of students consider it as unhealthy while $13 \%$ considered as healthy while in Fig 2 depicts $60 \%$ of individual agree eating on daily basis makes them eat more.

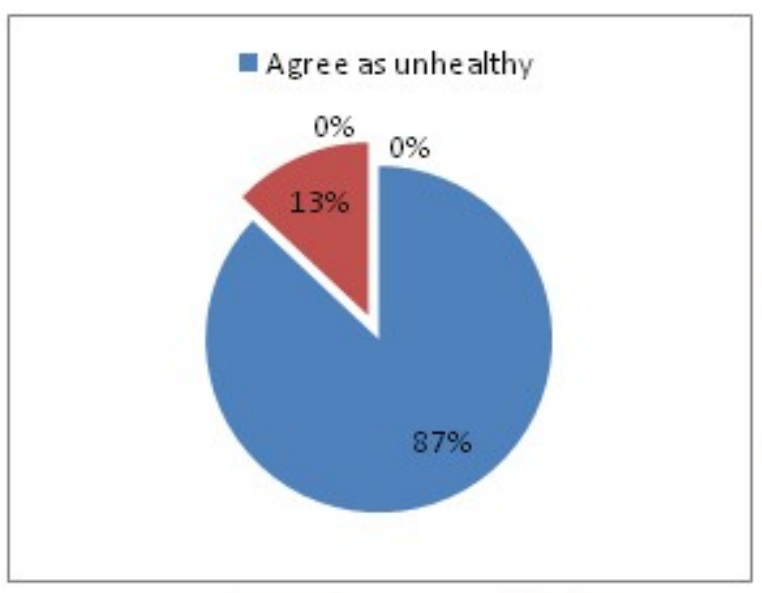

\section{Impact of Banning}

The impact of the recent ban made on one of the most popular fast food, maggi noodles the adolescents reposes were, $67 \%$ agree that it is necessary to ban these foods due to uncontrolled use of chemicals. 50\% responded that this ban has influenced the junk food consumption. Also this ban resulted $80 \%$ of respondents to change their pattern of eating food to healthy one. $90 \%$ of individuals consider it as unsafe to take similar kinds of foods as it may also contain harmful substances in large quantities.

$87 \%$ of individuals said that their parents restricts strictly from taking this type of food after this banning.

Other than there 21 questions 2 questions were asked generally whether the consumption pattern among new generation will increase tremendously in coming years and will it affect once growing society, the response was so unanimous as they all agreed that it will affect the society.

\section{CONCLUSION}

For majority of the adolescents though the frequency of visit is comparatively less, whenever they go only prefer to have these junk foods. Many of them made it as an alternative to breakfast.

Taste and appearance together are the most tempting factors for adolescents to have these junk foods. Other factors like time, conveniences, peer group, emotions etc. also plays a good role in this influence of junk foods.

According to the study done the knowledge and awareness about the nutritional aspects, the additives 
and their safety level, Quality etc. is very poor in majority of the adolescents.

Gracefully majority have an attitude that junk foods are unhealthy even if they have it regularly and they prefer junk foods than other nutritionally better foods. As this present study also focused to find the impact of the ban made on these junk foods, the result showed both children as well as parents have great change in their attitude and eating habit. Majority have changed their pattern of eating food to a healthy diet. Moreover parents restrict their children from having it.

As per the study it can be concluded that the influence of junk foods among adolescents is high and increasing day by day due to many of its characteristics such as taste, appearance, peer group etc. But hopefully the study showed that the recent ban made on this due to the lead content in it have altered this trend to an appreciable level. The awareness about health aspects and the side effects of these types of foods can reduce the fashion of junk food among adolescents.

\section{REFERENCES}

1) Kaushik, J.S., M. Narang and A. Parakh, 2011. Fast food consumption in children. Indian Pediatrics, 48: 97-101.

2) Habib, Q.F., R.A. Dardak and S. Zakaria, 2011. Consumer's preference and consumption towards fast food: Evidences from Malaysia. Bus. Manage. Q. Rev., 2: 14-27.

3) Bowman, S.A., S.L. Gortmaker, M.A. Pereira and D.S. Ludwig, 2004. Effects of fast-food consumption on energy intake and diet quality among children in a national household survey. Pediatrics, 113: 112-118.

4) Guthrie, J., B.H. Lin and E. Frazao, 2002. Role of food prepared away from home in American diet, 1977-78 versus 1994-96: Changes and consequences. J. Nutr. Educ. Behav., 34: 140-150. 